\title{
NOUVELle
}

\section{Des complexes protéiques impliqués dans la surveillance de I'ARN nucléaire inhibent la transcription du VIH-1}

\author{
Kader Salifou, Rosemary Kiernan, Xavier Contreras
}

Institut de génétique humaine, UMR 9002, CNRS, Université de Montpellier, Gene regulation laboratory, 141, rue de la Cardonille, 34396 Montpellier, France.

xavier.contreras@igh.cnrs.fr rosemary.kiernan@igh.cnrs.fr

de l'expression du génome viral [5]. L'exosome nucléaire est un complexe protéique jouant un rôle crucial dans la maturation des ARN (dont les ARN ribosomiques) et l'élimination des ARN aberrants [6]. Nous avions en effet montré que l'inhibition de l'expression de sa principale sous-unité catalytiques, RRP6 (ribosomal RNA-processing protein 6), induisait une forte augmentation de la transcription provenant d'un promoteur VIH-1. Cette inhibition résultait de la dégradation de l'ARN viral naissant par RRP6.

Plus récemment, dans un manuscrit paru dans PLOS Pathogens [7], nous avons caractérisé les protéines associées à RRP6 et à son principal partenaire, MTR4 (exosome RNA helicase). Nous avons pu montrer qu'il existe majoritairement deux complexes de l'exosome nucléaire bien distincts, contenant chacun RRP6 et MTR4, et caractérisés par la présence respective des protéines à doigts de zinc ZCCHC8 (zinc finger CCHC domaincontaining protein 8) et $\mathrm{ZFC3Hl} \mathrm{(zinc}$ finger (3HI-type containing). Des expériences d'immunoprécipitation nous ont permis de montrer que ces protéines interagissaient physiquement avec l'ARN polymérase 2. Par immunoprécipitation de chromatine (ChIP [chromatin immunoprecipitation]) et $A R N-C h I P$, nous avons également montré que $\mathrm{ZFC} 3 \mathrm{Hl}$ et ZCCHC8 sont associés directement avec la chromatine du promoteur du VIH, avec un pic d'intensité près du point $+l$ de transcription, suggérant une implication dans les étapes précoces de la transcription du virus. Enfin, il est impor- 


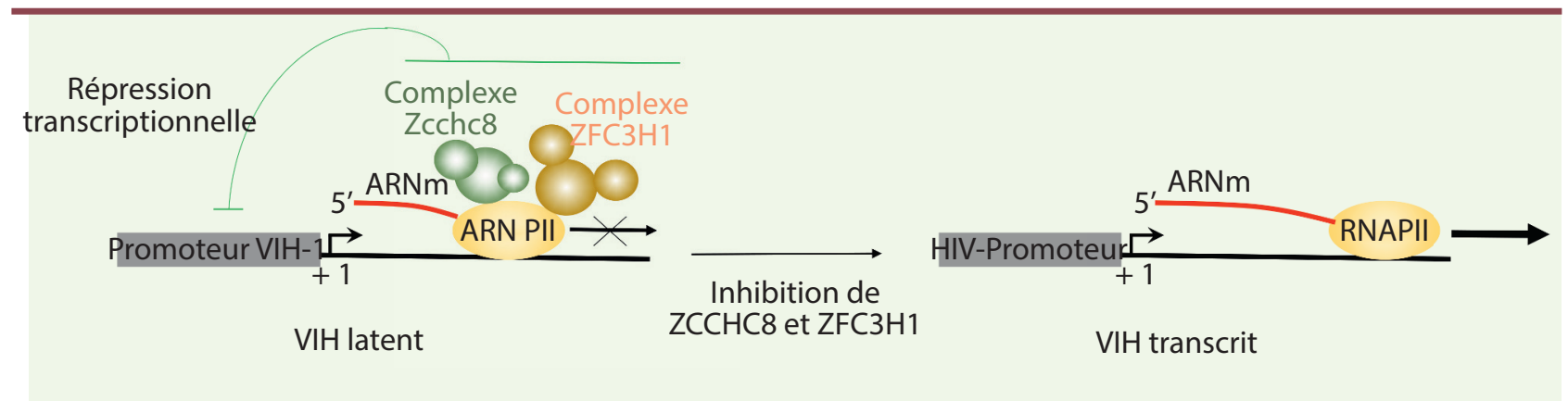

Figure 1. L'inhibition de ZCCHC8 et ZFC3HI permet d'activer la transcription du VIH-1 latent. Les deux complexes de l'exosome nucléaire contenant respectivement $\mathrm{ZFC3H1} \mathrm{(zinc} \mathrm{finger} \mathrm{CCHC} \mathrm{domain-containing} \mathrm{protein} \mathrm{8)} \mathrm{et} \mathrm{ZCCHC8} \mathrm{(zinc} \mathrm{finger} \mathrm{C3H1-type} \mathrm{containing)} \mathrm{sont} \mathrm{associés} \mathrm{avec} \mathrm{l'ARN}$ polymérase 2 (ARN PII) et répriment la transcription à partir du promoteur du VIH-1 (virus de l'immunodéficience humaine de type 1). L'inhibition de l'expression de ces facteurs induit une activation de la transcription du virus latent. ARNm : ARN messagers.

tant de souligner que seule la déplétion concomitante des deux protéines a permis d'activer la transcription du promoteur VIH-1 à un niveau comparable à celui des cellules n'exprimant plus RRP6. En revanche, l'extinction unique de ZCCHC8 ou de ZFC3HI n'a pas eu d'effet majeur. Il existerait donc une compensation entre les deux protéines pour assurer la répression transcriptionnelle du VIH- 1 . Ces résultats montrent donc l'existence de deux complexes de l'exosome caractérisés respectivement par ZCCHC8 et ZFC3Hl qui répriment, indépendamment l'un de l'autre, la transcription du VIH1. L'interaction physique avec I'ARN polymérase ainsi que le recrutement au niveau du point +1 de transcription suggèrent fortement que ces complexes interviennent dans les étapes précoces de la transcription du virus.

\section{Les deux complexes majeurs de l'exosome nucléaire coopèrent pour réprimer le VIH latent}

Pour étudier la latence du virus, nous avons utilisé deux modèles cellulaires contenant un génome viral presque complet: des cellules J-Lat dérivées de la lignée lymphocytaire Jurkat (J-Lat) ; et des cellules primaires (des lymphocytes $\mathrm{T} C D 4^{+}$isolés d'individus sains) que nous avons infectées avec des formes modifiées du VIH-l.

Les cellules J-lat sont obtenues par la transduction de cellules Jurkat avec des particules virales contenant un génome quasi-complet (uniquement dépourvu de la protéine d'enveloppe et de la protéine Nef [negative regulatory factor]) et qui codent également la GFP (green fluorescent protein). L'expression de la GFP dans ces cellules dépend de la réactivation transcriptionnelle du virus, comme cela se produit dans les cellules exprimant le réservoir viral. Nous avons montré que la déplétion de MTR4 seule, ou de ZFC3Hl et ZCCHC8 en association, entraînait l'activation de l'expression de la GFP dans un nombre important de cellules. Couplée à un traitement des cellules par la trichostatine A (TSA), un inhibiteur d'histones déacétylases utilisé couramment pour induire l'activation transcriptionnelle dans ce système, la co-déplétion de ZFC3Hl et ZCCHC8 entraîne une activation transcriptionnelle plus importante. Le second modèle de latence que nous avons utilisé a été décrit récemment. II est fondé sur l'utilisation de lymphocytes $\mathrm{T} \mathrm{CD}^{+}$primaires infectés par un vecteur VIH-l modifié qui exprime deux protéines fluorescentes rapportrices, la GFP et la $m$ Cherry (protéine fluorescente rouge monomérique) [8]. La GFP est exprimée sous le contrôle du promoteur du VIH-I et remplace les gènes codant Nef et la protéine d'enveloppe. Elle permet donc de suivre l'expression du génome viral. La protéine mCherry est sous le contrôle d'un promoteur constitutif indépendant, EFl-alpha (human elongation factor-1 alpha); elle permet de sélectionner les cellules infectées. Les cellules infectées latentes n'expriment donc que la protéine mCherry, alors que les cellules infectées de façon productive expriment les deux protéines fluorescentes mCherry et GFP. II est ainsi possible de les différencier par une analyse en cytométrie de flux. Les cellules n'exprimant que la protéine mCherry (donc latentes) ont ainsi été triées puis incubées en présence de vecteurs lentiviraux exprimant des shRNA (short hairpin RNA) qui inhibent l'expression de MTR4 ou celles de $\mathrm{ZFC} 3 \mathrm{Hl}$ et ZCCHC8. L'inhibition de l'expression de ces facteurs s'est accompagnée de l'apparition de cellules doublement positives (pour la GFP et la protéine mCherry) traduisant une activation de la transcription du génome viral dans une partie de la population latente.

L'ensemble de nos travaux montre donc l'existence de deux complexes distincts de l'exosome nucléaire qui répriment la transcription du VIH-1 (Figure 1, partie gauche). L'inhibition de ces complexes contribue à ré-activer le virus latent qui est la barrière principale à l'éradication du VIH-1 (Figure 1, partie droite). Ces travaux s'inscrivent dans l'optique d'une meilleure compréhension des mécanismes de latence pour ouvrir la voie à de nouvelles pistes thérapeutiques qui pourraient conduire à l'éradication du VIH-l chez les patients infectés. $\diamond$ Nuclear RNA surveillance complexes control HIV-1 transcription 


\section{REMERCIEMENTS}

L'étude a été réalisée grâce aux financements suivants : ERC RNAMEDTGS, MSDAVENIR HIDEINFLAME\&SEQ, l'Agence Nationale de Recherches sur le Sida et les hépatites virales (ANRS) et Sidaction pour RK.

\section{LIENS D'INTÉRÊT}

Les auteurs déclarent n'avoir aucun lien d'intérêt concernant les données publiées dans cet article.

\section{RÉFÉRENCES}

1. Barré-Sinoussi F. L'infection VIH/sida : I'histoire exemplaire d'une épidémie qui résiste. Med Sci (Paris)
$2018 ; 34: 499-500$.

2. Schwartz C, Le Douce V, Cherrier T, et al. Un virus tapi dans l'ombre : les bases moléculaires de la latence du VIH-1. Partie I : la physiologie de la latence du VIH-1. Med Sci (Paris) $2010 ; 26: 159-64$.

3. Cherrier T, Le Douce V, Redel L, et al. Un virus tapi dans l'ombre : les bases moléculaires de la latence du VIH1. Partie II : la réactivation de la latence du VIH-l et ses implications thérapeutiques. Med Sci (Paris) 2010 ; 26:291-6.

4. Mbonye U, Karn J. The molecular basis for human immunodeficiency virus latency. Annu Rev Virol 2017 $4: 261-85$.

5. Wagshcal A, Rousset $\varepsilon$, Basavarajaiah P, et al. Microprocessor, Setx, Xrn2 and Rrp6 co-operate to induce premature termination of transcription by
RNAPII. Cell $2012 ; 150: 1147-57$

6. Lykke-Andersen S, Tomecki R, Jensenn TH, et al. The eukaryotic RNa exosome: same scaffold but variable catalytic subunits. RNA Biol 2011 ; 8: 61-6.

7. Contreras X, Salifou K, Sanchez G, et al. Nuclear RNA surveillance complexes silence HIV-1 transcription. PLoS Pathog 2018; 14 : e1006950.

8. Chavez L, Calvanese V, Verdin $\varepsilon$. HIV latency is established directly and early in both resting and activated primary CD4 T cells. PLoS Pathog $2015 ; 11$ : el004955.

\section{NOUVELLع}

\section{Le cholestérol cellulaire, un régulateur important de la douleur inflammatoire}

Patrick Delmas ${ }^{1}$, Françoise Padilla ${ }^{1}$, Corinne Poilbout ${ }^{2}$

\author{
${ }^{1}$ SomatoSens, Laboratoire de Neurosciences Cognitives, \\ UMR 7291 CNRS, Aix-Marseille-Université, CS80011, \\ boulevard Pierre Dramard, 13344 Marseille, France. \\ ${ }^{2}$ Centre de Psychiatrie et Neurosciences, \\ 102-108, rue de la Santé, 75014 Paris France. \\ patrick.delmas@univ-amu.fr
}

> Le cholestérol est probablement la molécule dont le nom est le plus familier du grand public. II a été découvert au XVIII ${ }^{e}$ siècle par François Poulletier de La Salle dans des calculs biliaires (d'où son nom, chole- [bile] et stereos [solide]), mais c'est surtout pour son implication comme facteur de risque dans les maladies cardio-vasculaires qu'il est connu. L'on sait moins que c'est un précurseur de nombreuses molécules de l'organisme et un composant majeur des membranes cellulaires animales.

Dans l'étude que nous avons publiée [1], nous montrons que le cholestérol membranaire joue un rôle important dans la régulation du message nociceptif et de la douleur inflammatoire, et qu'il pourrait bien trouver une réhabilitation, comme antalgique topique.

\section{Bon ou mauvais cholestérol ? Portrait- robot}

Le cholestérol est un lipide de la famille des stérols ; c'est une molécule amphiphile, avec une partie hydro- phile (ou tête polaire) et une partie hydrophobe (ou apolaire). Il est présent dans la totalité des tissus des vertébrés, en particulier dans le foie, le cerveau et la moelle épinière. II est en grande partie d'origine endogène, environ $70 \%$, avec une synthèse essentiellement hépatique; la part exogène étant issue de l'alimentation. C'est le foie qui est chargé de son élimination, d'où une nécessaire double circulation dans le corps, par voie sanguine. De par sa nature globalement hydrophobe, le cholestérol est insoluble dans le sang ; il est donc associé à des lipoprotéines pour son transport. Ces lipoprotéines sont classées selon leur densité : les LDL (low-density lipoprotein) emportent le cholestérol du foie vers les tissus périphériques et peuvent, en cas d'excès, former des plaques athérosclérotiques; les HDL (high-density lipoprotein) permettent le transport centripète du cholestérol vers le foie pour son élimination [2]. C'est par simplification que l'on parle donc de bon ou mauvais cholestérol, pour les taux sanguins de HDL vs LDL.

\section{Le cholestérol dans les membranes cellulaires}

Le cholestérol est impliqué dans la synthèse de nombreuses molécules stéroïdiennes [3]: les sels biliaires qui sont des dérivés polaires du cholestérol ; les hormones stéroïdiennes sexuelles, comme la progestérone, la testostérone et les œstrogènes; les glucocorticoïdes (cortisone, cortisol) ; et les minéralocorticoïdes (aldostérone). Toutes ces molécules ont pour précurseur le cholestérol. Il en va de même de la vitamine D. Le cholestérol est donc un élément fondamental pour de nombreuses fonctions de régulation hormonale et $d u$ métabolisme.

La majorité du cholestérol est cependant cellulaire: il se trouve dans les membranes plasmiques où il représente 30 à $45 \%$ des lipides. Grâce à ses propriétés amphiphiles, le cholestérol s'insère dans la bicouche lipidique des mem- 